\title{
Damping Optimization of Viscoelastic Laminated Sandwich Structures using the Direct Multisearch Method
}

\author{
J. F. Aguilar Madeira ${ }^{1,2}$, A.L. Araújo ${ }^{1}$ \\ C.M. Mota Soares ${ }^{1}$ and C.A. Mota Soares ${ }^{1}$ \\ ${ }^{1}$ IDMEC/IST-Instituto Superior Técnico \\ Technical University of Lisbon, Portugal \\ ${ }^{2}$ ISEL, Instituto Superior de Engenharia de Lisboa, Portugal
}

\begin{abstract}
A multiobjective approach for optimization of passive damping for vibration reduction in sandwich structures is presented in this paper. A layerwise finite element model for sandwich plates with viscoelastic core and anisotropic laminated face layers is used along with the complex modulus approach and the dynamic problem is solved in the frequency domain. Constrained optimization is conducted for maximisation of modal loss factors and minimisation of weight of sandwich beams and plates with elastic laminated constraining layers and a viscoelastic core, with layer thickness and laminate layer ply orientation angles as design variables. The problem is solved using the Direct MultiSearch (DMS) solver for derivative-free multiobjective optimization and solutions are compared with alternative ones obtained using genetic algorithms. DMS is a solver for multiobjective optimization problems which does not use any derivatives of the objective functions. It is based on a novel technique called direct multisearch, developed by extending direct search from single to multiobjective optimisation.
\end{abstract}

Keywords: multiobjective optimisation, direct multisearch, sandwich structures, damping.

\section{Introduction}

Structural damping can be defined as the process by which a structure or structural component dissipates mechanical energy or transfers it to connected structures or ambient media. These mechanisms have the effect of controlling the amplitude of resonant vibrations and modifying wave attenuation and sound transmission properties, increasing structural life through reduction in structural fatigue.

Passive damping treatments are widely used in engineering applications in order to reduce vibration and noise radiation $[1,2]$. Passive layer damping, usually imple- 
mented as constrained layer damping, is the most common form of damping treatment, where the damping layer deforms in shear mode, thus dissipating energy in a more efficient way.

The theoretical work on constrained layer damping can be traced to [3] and [4] for the axial and bending vibration of sandwich beams. Since then, different formulations and techniques have been reported for modeling and predicting the energy dissipation of the viscoelastic core layer in a vibrating passive constrained layer damping structure $[5,6,7]$. Other proposed formulations include thickness deformation of the core layer dealing with the cases where only a portion of the base structure receives treatment [8].

Optimal design of constrained layer damping treatments of vibrating structures has been a main subject of research, aiming at the maximisation of modal damping ratios and modal strain energies, by determining the optimal material and geometric parameters of the treatments, or minimising weight by selecting their optimal length and location. For example, [9] optimized performance of constrained layer damping treatments by selecting the optimal thickness and shear modulus of the viscoelastic layer, and $[10,11]$ used a genetic algorithm and beam finite elements to maximise the damping factor for partially treated beams, using as design variables the dimensions and locations of the patches. As verified by [12], this layout optimization can lead to significant saving in the amount of material used. For fully covered sandwich beams, [13] determined the optimal passive constrained layer damping, with layer thicknesses as design variables. The vibration damping of fully covered constrained layer damping structures is determined by a large number of parameters which include material properties and thicknesses of both the constraining layers and the viscoelastic layer. Damping optimisation of sandwich plates and beams with viscoelastic core material and composite laminated face layers has been conducted recently by Araújo et al $[14,15]$, where the design variables are layer thicknesses and fibre orientation. In these works, modal loss factors are maximised subject to displacement, failure and mass constraints. A multiobjective approach is also considered [14] for minimising weight and maximising the fundamental modal loss factor of the sandwich. More recently, damping optimisation was considered in the framework of both active and passive damping [16].

A constrained nonlinear multiobjective optimization problem can be mathematically formulated as

$$
\begin{array}{cl}
\min & F(x) \equiv\left(f_{1}(x), f_{2}(x), \ldots, f_{m}(x)\right)^{\top} \\
\text { s. t. } & x \in \Omega
\end{array}
$$

involving $m$ objective functions $f_{j}: \Omega \subseteq \mathbb{R}^{n} \rightarrow \mathbb{R} \cup\{+\infty\}, j=1, \ldots, m$ to minimize. Recall that to maximise $f_{j}$ is equivalent to minimise $-f_{j}$. $\emptyset \neq \Omega \subseteq \mathbb{R}^{n}$ represents the feasible region.

In the presence of $m(\geq 2)$ objective functions, the minimiser of one function are not necessarily the minimiser of another. In this case, we don't have a single point 
that yields the "optimum point for all objectives". Instead, we have a set of points, called Pareto optimal or nondominated set. Given two points $x_{1}, x_{2}$ in $\Omega, x_{1}$ is said to dominate, in Pareto sense, another point $x_{2}$ if and only if solution $x_{1}$ is strictly better than $x_{2}$ in at least one of the objectives and point $x_{1}$ is not worse than $x_{2}$ in any of the objectives. A set of points in $\Omega$ is nondominated when no point in the set is dominated by another one in the set.

Direct MultiSearch (DMS) [17] is a solver for multiobjective optimization problems, without the use of derivatives and does not aggregate any components of the objective function. It essentially generalizes all direct-search methods of directional type from single to multiobjective optimization. DMS maintains a list of feasible nondominated points. At each iteration, the new feasible evaluated points are added to this list and the dominated ones are removed. Successful iterations correspond then to an iterate list changes, meaning that a new feasible nondominated point was found. Otherwise, the iteration is declared as unsuccessful.

In this work, an application of the Direct MultiSearch (DMS) [17] solver for derivative-free multiobjective optimisation is made to the problems addressed in Araújo et al. [14] for maximisation of modal loss factors and minimisation of weight of sandwich plates with elastic laminated constraining layers and a viscoelastic core. The obtained Pareto front solutions are compared with the ones presented in the literature.

The paper is organised as follows. In section 2 the finite element model for composite laminated sandwich plates with viscoelastic core is presented. In section 3 the different objective functions are shown along with the design constraints. Section 4 presents two applications, one for a sandwich beam and another one for a sandwich plate. We finish the article with some conclusions and remarks on the obtained solutions and the methodology used.

\section{Sandwich finite element model}

We will use a layerwise finite element model [18] to analyze sandwich laminated plates with a viscoelastic $(v)$ core and laminated anisotropic face layers $\left(e_{1}, e_{2}\right)$, and top and bottom piezoelectric sensor $(s)$ and actuator (a) layers, as shown in Figure 1. Although sensor and actuator layers are included in the formulation presented here, the piezoelectric effect will not be considered in this work, as it deals only with passive damping. Hence, these sensor and actuator layers will have a purely elastic behaviour.

The basic assumptions in the development of the sandwich plate model are: all points on a normal to the plate have the same transverse displacement $w(x, y, t)$, where $t$ denotes time, and the origin of the $z$ axis is the medium plane of the core layer; no slip occurs at the interfaces between layers; the displacement is $C^{0}$ along the interfaces; elastic layers $\left(a, e_{2}, e_{1}, s\right)$ are each modelled with first order shear deformation theory (FSDT) and viscoelastic core $(v)$ with a higher order shear deformation theory (HSDT); all materials are linear, homogeneous and orthotropic and the elastic layers $\left(e_{1}\right)$ and $\left(e_{2}\right)$ are made of laminated composite materials; for the viscoelastic core, 


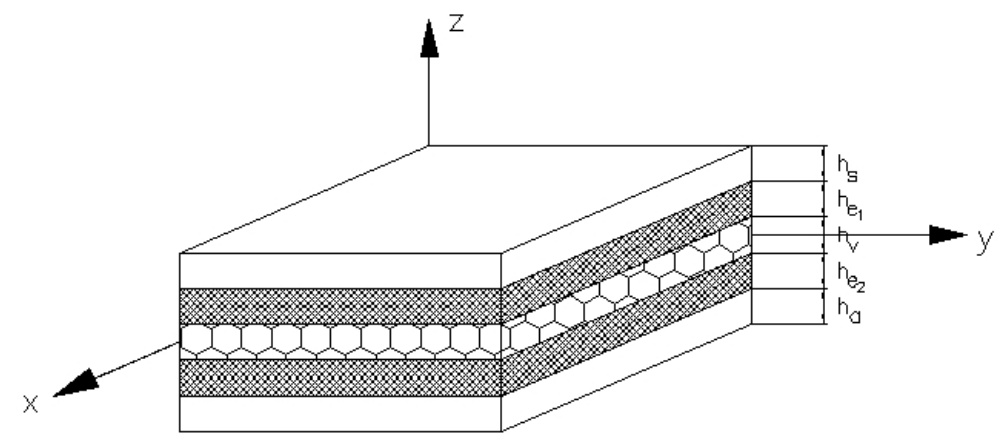

Figure 1: Sandwich plate.

material properties are complex and frequency dependent.

The FSDT displacement field of the face layers may be written in the general form:

$$
\begin{aligned}
& u^{i}(x, y, z, t)=u_{0}^{i}(x, y, t)+\left(z-z_{i}\right) \theta_{x}^{i} \\
& v^{i}(x, y, z, t)=v_{0}^{i}(x, y, t)+\left(z-z_{i}\right) \theta_{y}^{i} \\
& w^{i}(x, y, z, t)=w_{0}(x, y, t)
\end{aligned}
$$

where $u_{0}^{i}$ and $v_{0}^{i}$ are the in-plane displacements of the mid-plane of the layer, $\theta_{x}^{i}$ and $\theta_{y}^{i}$ are rotations of normals to the mid-plane about the $y$ axis (anticlockwise) and $x$ axis (clockwise), respectively, $w_{0}$ is the transverse displacement of the layer (same for all layers in the sandwich), $z_{i}$ is the $z$ coordinate of the mid-plane of each layer, with reference to the core layer mid-plane $(z=0)$, and $i=a, e_{2}, e_{1}, s$ is the layer index.

For the viscoelastic core layer, the HSDT displacement field is written as a second order Taylor series expansion of the in-plane displacements in the thickness coordinate, with constant transverse displacement:

$$
\begin{aligned}
& u^{v}(x, y, z, t)=u_{0}^{v}(x, y, t)+z \theta_{x}^{v}+z^{2} u_{0}^{* v}+z^{3} \theta_{x}^{* v} \\
& v^{v}(x, y, z, t)=v_{0}^{v}(x, y, t)+z \theta_{y}^{v}+z^{2} v_{0}^{* v}+z^{3} \theta_{y}^{* v} \\
& w^{v}(x, y, z, t)=w_{0}(x, y, t)
\end{aligned}
$$

where $u_{0}^{v}$ and $v_{0}^{v}$ are the in-plane displacements of the mid-plane of the core, $\theta_{x}^{v}$ and $\theta_{y}^{v}$ are rotations of normals to the mid-plane of the core about the $y$ axis (anticlockwise) and $x$ axis (clockwise), respectively, $w_{0}$ is the transverse displacement of the core (same for all layers in the sandwich). The functions $u_{0}^{* v}, v_{0}^{* v}, \theta_{x}^{* v}$ and $\theta_{y}^{* v}$ are higher order terms in the series expansion, defined also in the mid-plane of the core layer.

Imposing displacement continuity conditions at the layer interfaces and retaining the rotational degrees of freedom of the elastic layers, while eliminating the corresponding in-plane displacement ones, the generalized displacement field has 17 unknowns: 


$$
\begin{array}{lllllllllllllllll}
\theta_{x}^{a} & \theta_{y}^{a} & \theta_{x}^{e_{2}} & \theta_{y}^{e_{2}} & u_{0}^{v} & v_{0}^{v} & w_{0} & \theta_{x}^{v} & \theta_{y}^{v} & u_{0}^{* v} & v_{0}^{* v} & \theta_{x}^{* v} & \theta_{y}^{* v} & \theta_{x}^{e_{1}} & \theta_{y}^{e_{1}} & \theta_{x}^{s} & \theta_{y}^{s}
\end{array}
$$

We consider that fiber-reinforced laminae in elastic multi-layers $\left(e_{1}\right)$ and $\left(e_{2}\right)$, sensor $(s)$ and actuator $(a)$ layers, and viscoelastic core $(v)$ are characterized as orthotropic. Constitutive equations for each lamina in the sandwich may then be expressed in the principal material directions $\left(x_{1}, x_{2}, x_{3}=z\right)$, and for the zero transverse normal stress situation, as [19]:

$$
\left\{\begin{array}{l}
\sigma_{11} \\
\sigma_{22} \\
\sigma_{23} \\
\sigma_{13} \\
\sigma_{12}
\end{array}\right\}=\left[\begin{array}{ccccc}
Q_{11} & Q_{12} & 0 & 0 & 0 \\
Q_{12} & Q_{22} & 0 & 0 & 0 \\
0 & 0 & Q_{44} & 0 & 0 \\
0 & 0 & 0 & Q_{55} & 0 \\
0 & 0 & 0 & 0 & Q_{66}
\end{array}\right]\left\{\begin{array}{c}
\varepsilon_{11} \\
\varepsilon_{22} \\
\gamma_{23} \\
\gamma_{13} \\
\gamma_{12}
\end{array}\right\}
$$

where $\sigma_{i j}$ are stress components, $\varepsilon_{i j}$ and $\gamma_{i j}$ are strain components, and $Q_{i j}$ are reduced stiffness coefficients. Expressions for the reduced quantities mentioned above can be found in [19]. For the viscoelastic core layer, the reduced stiffness coefficients $Q_{i j}$ are complex quantities, since the complex modulus approach was used in this work, using the elastic-viscoelastic principle. In this case, the usual engineering moduli may be represented by complex quantities:

$$
\begin{aligned}
& E_{1}(i \omega)=E_{1}^{\prime}(\omega)\left(1+i \eta_{E_{1}}(\omega)\right) \\
& E_{2}(i \omega)=E_{2}^{\prime}(\omega)\left(1+i \eta_{E_{2}}(\omega)\right) \\
& G_{12}(i \omega)=G_{12}^{\prime}(\omega)\left(1+i \eta_{G_{12}}(\omega)\right) \\
& G_{23}(i \omega)=G_{23}^{\prime}(\omega)\left(1+i \eta_{G_{23}}(\omega)\right) \\
& G_{13}(i \omega)=G_{13}^{\prime}(\omega)\left(1+i \eta_{G_{13}}(\omega)\right) \\
& \nu_{12}(i \omega)=\nu_{12}^{\prime}(\omega)\left(1-i \eta_{\nu_{12}}(\omega)\right)
\end{aligned}
$$

where the prime quantities denote storage moduli, associated material loss factors are represented by the letter $\eta, \omega$ represents frequency of vibration and $i=\sqrt{-1}$ is the imaginary unit. Furthermore, in Equation (5), E, $G$ and $\nu$ denote Young's moduli, shear moduli and Poisson's ratio, respectively.

The definition of constitutive relations of a laminate is usually made in terms of stress resultants. These forces and moments are defined separately for the viscoelastic core and the elastic layers [20].

The equations of motion for the plate are obtained by applying Hamilton's principle, using an eight node serendipity plate element with 17 degrees of freedom per node:

$$
\mathbf{M u}+\mathbf{K u}=\mathbf{f}
$$


where $\mathbf{u}$ and $\ddot{\mathbf{u}}$ are generalised displacement degrees of freedom and corresponding accelerations, respectively, $\mathbf{M}$ and $\mathbf{K}$ are the mass and stiffness matrices, respectively, and $\mathbf{f}$ is the externally applied load vector. One should note that the viscoelastic behaviour of the core translates into a complex and frequency dependent stiffness matrix K.

Assuming harmonic vibrations, the forced vibration problem is solved in the frequency domain, which implies the solution of the following linear system of equations for each frequency point:

$$
\left[\mathbf{K}(\omega)-\omega^{2} \mathbf{M}\right] \mathbf{u}(\omega)=\mathbf{F}(\omega)
$$

where $\mathbf{F}(\omega)=\mathcal{F}(\mathbf{f}(t))$ is the Fourier transform of the time domain force history $\mathbf{f}(t)$.

For the free vibration problem, Equation (7) reduces to the following non-linear complex eigenvalue problem:

$$
\left[\mathbf{K}(\omega)-\lambda_{n}^{*} \mathbf{M}\right] \mathbf{u}_{n}=\mathbf{0}
$$

where, the complex eigenvalue $\lambda_{n}^{*}$ is written as:

$$
\lambda_{n}^{*}=\lambda_{n}\left(1+i \eta_{n}\right)
$$

and $\lambda_{n}=\omega_{n}^{2}$ is the real part of the complex eigenvalue and $\eta_{n}$ is the corresponding modal loss factor.

The non-linear eigenvalue problem is solved iteratively using ARPACK [21] with a shift-invert transformation. The iterative process is considered to have converged when:

$$
\frac{\left\|\omega_{i}-\omega_{i-1}\right\|}{\omega_{i-1}} \leq \epsilon
$$

where $\omega_{i}$ and $\omega_{i-1}$ are current and previous iteration values for the real part of the particular eigenfrequency of interest, respectively, and $\epsilon$ is the convergence tolerance.

\section{Optimal design formulation}

The objective of this study is to maximise damping in sandwich plate structures while simultaneously minimising weight, which are conflicting objectives. If the structure is subjected to a given load or load set, design constraints such as maximum displacement, stress failure criteria, as well as physical constraints on design variables and objective function should be imposed.

For damping maximisation with passive treatments in sandwich type structures, the overall goal will be to maximise the fundamental modal loss factor $\eta_{1}$, while at the same time minimising the weight or mass $m$ of the structure: 


$$
\begin{array}{ll}
\min _{x} & F(x)=\left(f_{1}(x), f_{2}(x)\right)^{T} \\
\text { s.t. } & g_{1}:-\eta_{1} \leq 0 \\
& g_{2}: \quad \frac{w}{w_{\max }}-1 \leq 0 \\
& g_{3}: \quad F_{T H}-1 \leq 0 \\
& x_{i}^{l} \leq x_{i} \leq x_{i}^{u}, \quad i=1, \ldots, n
\end{array}
$$

where $f_{1}=1 / \eta_{1}, f_{2}=M$ (mass), $w$ and $w_{\max }$ are the maximum displacement of the structure and the maximum allowable value of the displacement, respectively, and $F_{T H}$ is the Tsai-Hill failure criteria parameter for the elastic composite material layers, defined as:

$$
\begin{aligned}
F_{T H} & =\left(\frac{\sigma_{11}}{X}\right)^{2}+\left(\frac{\sigma_{22}}{Y}\right)^{2}+\left(\frac{\sigma_{33}}{Z}\right)^{2} \\
& -\left(\frac{1}{X^{2}}+\frac{1}{Y^{2}}-\frac{1}{Z^{2}}\right) \sigma_{11} \sigma_{22} \\
& -\left(\frac{1}{Y^{2}}+\frac{1}{Z^{2}}-\frac{1}{X^{2}}\right) \sigma_{22} \sigma_{33} \\
& -\left(\frac{1}{Z^{2}}+\frac{1}{X^{2}}-\frac{1}{Y^{2}}\right) \sigma_{11} \sigma_{33} \\
& +\left(\frac{\sigma_{23}}{R}\right)^{2}+\left(\frac{\sigma_{13}}{S}\right)^{2}+\left(\frac{\sigma_{12}}{T}\right)^{2}<1
\end{aligned}
$$

where the stress components $\sigma_{i j}$ are calculated for each elastic layer ply and refer to the principal material directions of the ply, $X, Y$ and $Z$ are lamina failure stresses in the associated principal directions, which must respect the sign of the stresses, and one must consider different values in traction and compression. $R, S$ and $T$ are failure stresses in shear for the associated planes in Equation (12).

Assuming a uniform sandwich plate structure made of a given set of materials, with fixed in-plane dimensions, the natural choice for the design variables $x_{i}$ in Equation (11) are the thicknesses of the constituent layers and the orientation angles of the laminated elastic composite material plies. In Equation (11), $x_{i}^{l}$ and $x_{i}^{u}$ are the lower and upper bounds on the design variables.

Calculation of the objective function is done by solving the eigenvalue problem of Equation (8) iteratively, for a frequency dependent complex stiffness matrix and real mass matrix.

Calculation of response quantities such as displacements and stresses are done after the eigenvalue problem has been solved. This problem is solved in the frequency domain, by first making a forward Fourier transform of the applied load time history, and then solving Equation (7) in order to the displacement vector, for the resonant 
frequency of interest. Afterwards, stresses in each elastic material layer ply are calculated and the Tsai-Hill factor $F_{T H}$ in Equation (12) is evaluated. The constraints $g_{1}$, $g_{2}$ and $g_{3}$ shown in Equation (11) are imposed by penalising the objective functions in case of constraint violation. The side constraints are applied directly in the DMS formulation.

\section{Applications}

For the applications described in this section, the elastic material properties for the composite material layers are: $E_{1}=98.0 \mathrm{GPa}, E_{2}=7.9 \mathrm{GPa}, \nu_{12}=0.28, \rho=$ $1520 \mathrm{~kg} / \mathrm{m}^{3}$, and for the isotropic viscoelastic core: $G=20(1+0.3 i) \mathrm{MPa}, \nu=0.49$, $\rho=1140 \mathrm{~kg} / \mathrm{m}^{3}$. As for the top and bottom isotropic layers, the elastic material properties are: $E=2.0 \mathrm{GPa}, \nu=0.29$ and $\rho=1800 \mathrm{~kg} / \mathrm{m}^{3}$.

The default parameters of DMS were used (version 0.2, May 2011) without cache.

\subsection{Passive design of a simply supported sandwich beam}

A simply supported sandwich beam of length $L=1 \mathrm{~m}$ and width $b=0.005 \mathrm{~m}$, with symmetric layout of layers is considered. The top and bottom isotropic elastic layers have a thickness of $h_{a}=h_{s}=0.1 \mathrm{~mm}$, the composite layers will have equal thickness $h_{e_{1}}=h_{e_{2}}$, considered as a design variable. As for the viscoelastic core, its thickness $h_{v}$ will also be a design variable. Thickness design variables are allowed to vary between $0.5 \mathrm{~mm}$ and $10 \mathrm{~mm}$. The orientation angles of the composite layers are considered to be constant at $0^{\circ}$ w.r.t. the longitudinal axis.

The fundamental flexural modal loss factor of the beam will be maximised, while minimising total mass with a maximum allowable displacement $w_{\max }=h / 5$, where $h=h_{a}+h_{e_{1}}+h_{v}+h_{e_{2}}+h_{s}$ is the total thickness of the beam. The failure stresses in the Tsai-Hill expression were considered to be, for the elastic layers, $X=820 \mathrm{MPa}$, $Y=Z=45 \mathrm{MPa}$, both in tension and compression, and $R=S=T=45 \mathrm{MPa}$. The excitation consisted of a $1 \mathrm{~N}$ force applied at the mid-point of the beam at $t=0$.

For the layerwise model, a $6 \times 1$ finite element model was used, with a total of 543 degrees of freedom.

The nondominated solutions for each algorithm are presented in Figure 2.

In Figure 2 the few solutions named GA were obtained by genetic algorithms in [14] and are compared with those obtained by DMS. We can observe that on the left portion of the Pareto front in Figure 2, both approaches give similar results while for the remaining part of the curve, DMS provides substantially better solutions. 


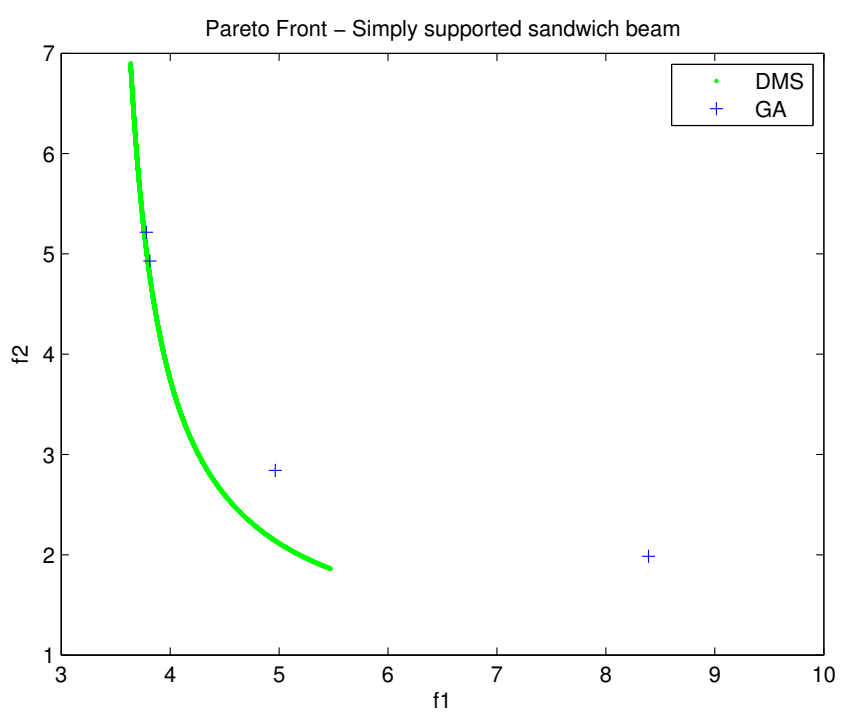

Figure 2: Pareto front for the simply supported sandwich beam. Mass $\left(f_{2}\right)$ in gram.

\subsection{Passive design of a simply supported sandwich plate}

A simply supported sandwich plate of in-plane dimensions $300 \mathrm{~mm} \times 200 \mathrm{~mm}$ with symmetric layout of layers is considered. The top and bottom elastic and isotropic layers have a thickness $h_{a}=h_{s}=0.1 \mathrm{~mm}$. The composite elastic layers are made of 3 plies each with equal thickness, which are design variables. As for the viscoelastic core, its thickness $h_{v}$ will also be a design variable. The thickness design variables can take values from $0.5 \mathrm{~mm}$ to $10 \mathrm{~mm}$. The orientation angles of the composite elastic layers are considered also to be design variables, assuming values between $0^{\circ}$ and $175^{\circ}$.

The fundamental flexural modal loss factor of the beam will be maximised, along with the minimisation of weight with a maximum allowable displacement $w_{\max }=$ $h / 5$, where $h=h_{a}+h_{e_{1}}+h_{v}+h_{e_{2}}+h_{s}$ is the total thickness of the plate. The failure stresses in Equation (12) are, for the elastic layers, $X=820 \mathrm{MPa}, Y=Z=45 \mathrm{MPa}$, both in tension and compression, and $R=S=T=45 \mathrm{MPa}$. The excitation consisted of a $10 \mathrm{~N}$ force applied at the mid-point of the plate at $t=0$.

For the layerwise model, a $6 \times 4$ finite element mesh was used, with a total of 1505 degrees of freedom.

The nondominated solutions for each algorithm are presented in Figure 3.

In Figure 3 the few solutions named GA were obtained by genetic algorithms in [14] and are compared with those obtained by DMS. We can observe that on the left and right portions of the Pareto front in Figure 3, both approaches give similar results while for the intermediate part of the curve, DMS provides slightly better solutions. When comparing the current Pareto front obtained by DMS with the one reported by Araújo at al. [14] we can conclude that DMS provides more and better solutions for 


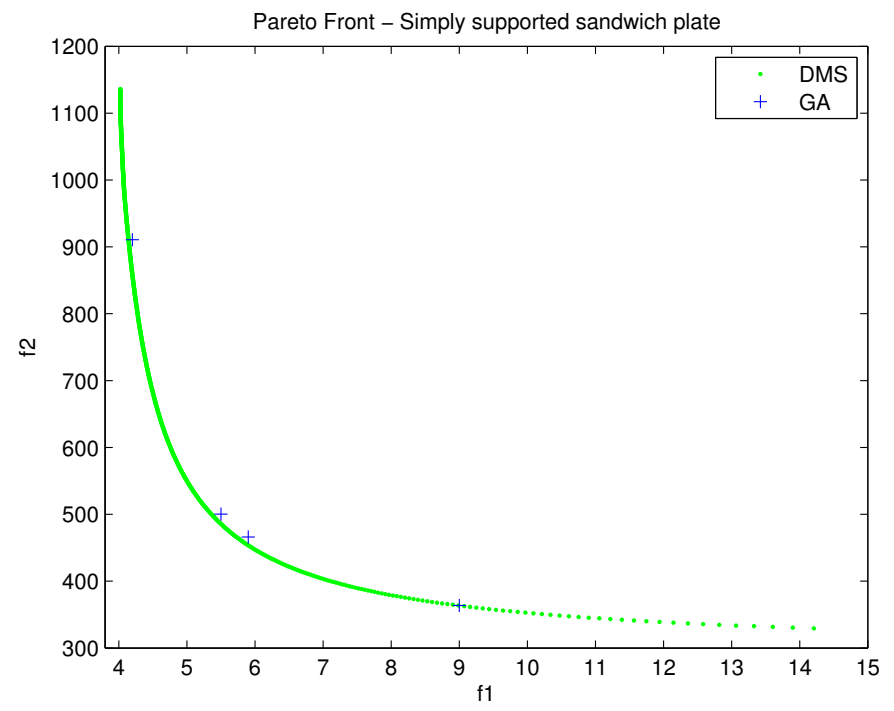

Figure 3: Pareto front for the simply supported sandwich plate. Mass $\left(f_{2}\right)$ in gram.

most cases.

\section{Conclusions}

In this paper we apply for the first time direct multisearch for the solution of a problem of passive damping optimization in viscoelastic sandwich composite structures. The solutions obtained herein were compared with previous solutions available in the literature and obtained by a genetic algorithm approach. We conclude that the present method provides more reliable solutions for the problem of maximising damping and simultaneously minimising weight.

\section{Acknowledgements}

The authors thank the financial support of Fundação para a Ciência e a Tecnologia, Portugal, for the financing of IDMEC in the framework of LAETA and through project PTDC/EME-PME/120830/2010.

\section{References}

[1] A. Nashif, D. Jones, J. Henderson, Vibration Damping, John Wiley \& Sons, New York, 1985. 
[2] C. Sun, Y. Lu, Vibration Damping of Structural Elements, Prentice Hall PTR, Englewood Cliffs, New Jersey, 1995.

[3] R. DiTaranto, "Theory of vibratory bending for elastic and viscoelastic layered finite-length beams", ASME Journal of Applied Mechanics, 32: 881-886, 1965.

[4] D. Mead, S. Markus, "The forced vibration of a three-layer, damped sandwich beam with arbitrary boundary conditions", AIAA Journal, 10: 163-175, 1969.

[5] D. Rao, "Frequency and loss factors of sandwich beams under various boundary conditions", International Journal of Mechanical Engineering Science, 20: 271-278, 1978.

[6] M. Yan, E. Dowell, “Governing equations of vibrating constrained-layer damping sandwich plates and beams", ASME Journal of Applied Mechanics, 39: 1041-1046, 1972.

[7] M. Rao, S. He, "Dynamic analysis and design of laminated composite beams with multiple damping layers", AIAA Journal, 31: 736-745, 1993.

[8] B. Douglas, J. Yang, "Transverse compressional damping in the vibratory response of elastic-viscoelastic beams", AIAA Journal, 16: 925-930, 1978.

[9] A. Baz, J. Ro, "Optimum design and control of active constrained layer damping”, Journal of Mechanical Engineering Design, 117: 135-144, 1995.

[10] J. Marcelin, P. Trompette, A. Smati, "Optimal constrained layer damping with partial coverage", Finite Elements in Analysis and Design, 12: 273-280, 1992.

[11] J. Marcelin, S. Shakhesi, F. Pourroy, "Optimal constrained layer damping of beams: experimental numerical studies", Shock and Vibration, 2: 445-450, 1995.

[12] D. Nokes, F. Nelson, "Constrained layer damping with partial coverage", Shock and Vibration Bulletin, 38: 5-10, 1968.

[13] J. Lifshitz, M. Leibowitz, "Optimal sandwich beam design for maximum viscoelastic damping", International Journal of Solids and Structures, 23: 10271034, 1987.

[14] A. Araújo, P. Martins, C.M. Soares, C.M. Soares, J. Herskovits, "Damping optimization of viscoelastic laminated sandwich composite structures", Structural and Multidisciplinary Optimisation, 39: 569-579, 2009.

[15] A. Araújo, C.M. Soares, C.M. Soares, J. Herskovits, "Optimal design and parameter estimation of frequency dependent viscoelastic laminated sandwich composite plates", Composite Structures, 92: 2321-2327, 2010.

[16] A. Araújo, P. Martins, C.M. Soares, C.M. Soares, J. Herskovits, "Damping optimisation of hybrid active-passive sandwich composite structures", Advances in Engineering Software, 46: 69-74, 2012.

[17] A.L. Custódio, J.F.A. Madeira, A.I.F. Vaz, L.N. Vicente, "Direct multisearch for multiobjective optimization", SIAM Journal of Optimization, 21: 1109-1140, 2011.

[18] A. Araújo, C.M. Soares, C.M. Soares, "Finite element model for hybrid activepassive damping analysis of anisotropic laminated sandwich structures", Journal of Sandwich Structures and Materials, 12(4): 397-419, 2010.

[19] A. Araújo, H. Lopes, M. Vaz, C.M. Soares, J. Herskovits, P. Pedersen, "Pa- 
rameter estimation in active plate structures", Computers and Structures, 84: 1471-1479, 2006.

[20] A. Araújo, C.M. Soares, J. Herskovits, P. Pedersen, "Development of a finite element model for the identification of mechanical and piezoelectric properties through gradient optimisation and experimental vibration data", Composite Structures, 58: 307-318, 2002.

[21] D. Sorensen, "Implicitly restarted Arnoldi/Lanczos methods for large scale eigenvalue calculations", Technical Report Technical Report TR95-13, Department of Computational and Applied Mathematics, Rice University, Houston, Texas, 1995. 